Draft of paper to appear in in Symons, J., and Calvo, P., eds., The Routledge Companion to the Philosophy of Psychology. Routledge, London, 2009.

\title{
Evolutionary Models In Psychology
}

\section{Introduction}

For anyone whose world-view is reliably informed by science, the idea that the complex organs of the human body should be explained scientifically as the product of evolution is, as they say, a no-brainer. And that includes the most complex organ of all, the human brain. But now what about the human mind? Is that to be explained scientifically as the product of evolution? Here it is worth beginning with what the psychologists Tooby and Cosmides (1992; more on them below) call the Standard Social Science Model (SSSM) of mind. According to the SSSM, the mind's innate evolutionary endowment (the cognitive elements with which we are born) is nigh on exhausted by our senses, some basic drives such as hunger and fear, and a capacity for general purpose learning. In other words, knowledge-wise the mind at birth is pretty much what philosophers will recognize as a Lockean blank slate. What evolution has done is give that epistemically empty vessel the means to learn post-natally from its cultural environment. At root, then, it's culture, rather than evolution, that explains the character of the complex information-rich structure which that empty vessel becomes.

Now, Tooby and Cosmides are not just psychologists, they are evolutionary psychologists, and their understanding of what an evolutionary model in psychology ought to look like is very different indeed from the SSSM-generated picture. Here we need to get clear about some labels. In the current intellectual climate, the term 'evolutionary psychology' is often used to identify not simply any psychological science that takes its cues from evolutionary biology, but rather a very specific, limelighthogging, socially explosive, scientifically controversial, and philosophically intriguing stream of such work. The research in question is based on a number of conceptual and theoretical principles (to be discussed later) that are not merely antithetical to the SSSM model of mind; in addition, they are rejected by plenty of other psychological theorists who take their work to have robust evolutionary roots. The narrow use of the term 'evolutionary psychology' is no doubt irksome to the latter group of thinkers, but (with suitable apologies) I shall adopt it in what follows. Moreover, given the fact that evolutionary psychology (narrowly conceived) has attracted a good deal of philosophical attention, both supportive and critical, I shall organize this entry around an attempt to lay bare exactly what the conceptual foundations of that specific paradigm are, plus a survey of some of the chief criticisms leveled against it. Certain other evolutionary models in psychology will make brief appearances as critical response to evolutionary psychology, which is not to say that this exhausts the interest of those alternative models.

\section{What is Evolutionary Psychology?}


One might usefully think of evolutionary psychology as being defined by two baseline commitments and three big ideas. Let's start with the baseline commitments. These come to light once we try to be more specific about what might be meant by the terms 'evolutionary' and 'psychology' in the moniker 'evolutionary psychology'.

Natural selection occurs whenever one has heritable variation in fitness, where fitness is understood as a measure of the capacity of an organism to reproduce in some environment. Since organisms exhibiting fitter traits are, on average, more likely to reproduce, if the fitness-bestowing traits are heritable, if there is competition for resources, and if the environment is stable enough, those traits will, over time, tend to spread though the population. Sexual selection is a variant of natural selection in which certain phenotypic features of one sex in a species evolve because the other sex prefers to mate with individuals who have those features; canonical examples include the male peacock's tail and deep voices in male humans. Darwin identified both non-sexual natural selection and sexual selection as evolutionary processes, and henceforth I shall group them together under the banner of Darwinian selection. Darwinian selection is a mechanism that results in the phenomenon of adaptation. Adaptation is the calibration of organisms to their environments, and the calibrated traits are called adaptations. In principle of course there could be other explanations for adaptation (God could have designed organisms that way) but adaptationists in evolutionary biology take it that where one genuinely has an adaptation one is looking at a product of Darwinian selection. It seems to most biologists that however one cuts the evolutionary cake, Darwinian selection will be at the centre of our understanding of evolution. For the evolutionary psychologist, however, it often seems that evolution simply is Darwinian selection; so the term 'evolutionary' in 'evolutionary psychology' just means 'explained by Darwinian selection'. This is the first baseline commitment of evolutionary psychology.

So what precisely is the explanatory target of Darwinian theorizing here? In the intellectual arena within which evolutionary psychology has emerged, some thinkers have pursued the following thought: if differences in human social behaviour result in differences in fitness, and if those behaviours are heritable, then selection can favour the fitter behaviour. This is the classical form of the discipline known as sociobiology (Wilson 1975). Classical sociobiologists attempt to explain behaviours such as rape, incest avoidance and male sexual promiscuity in terms of the fitness benefits that those behaviours might bestow under certain conditions. Thus Thornhill and Thornhill (1992) argue (controversially, as many critical responses at the time made clear) that rape by human males is an adaptation to sexual exclusion.

Ignoring empirical objections to particular models, is there anything wrong in principle with classical sociobiology? One big worry concerns the massive diversity of social behaviours that human beings perform - from culture to culture and from individual to individual. If human social behaviours are species-wide adaptations, the result of a long process of cumulative selection, one might expect those behaviours to be robust across different cultures. However, this is not the pattern we see in human cultural life. Human social behaviours seem extraordinarily sensitive to cultural factors, in a way that goes well beyond what could possibly be absorbed by the idea of adaptations that are 
expressed only in certain circumstances. And a similar point could be made about behavioural variations between individuals.

One response to this difficulty might be to run back into the arms of SSSM. But there is an alternative. Enter evolutionary psychology, which shifts the focus of Darwinian selective attention away from behaviours and onto the inner, neurally realized, psychological mechanisms that are the proximal causes of those behaviours. On this view, then, what gets selected for are not the behaviours themselves, but rather the psychological mechanisms that generate and control them. Just as there are anatomical adaptations (bodily structures shaped by natural selection to solve certain adaptive problems), there are psychological adaptations (cognitive structures shaped by natural selection to solve certain other adaptive problems). Thus, as Cosmides and Tooby (1987, 282) put it, '[the] evolutionary function of the human brain is to process information in ways that lead to adaptive behavior'.

We have now unearthed our second baseline commitment of evolutionary psychology, which concerns the 'psychology' part of the term. Evolutionary psychology is, as we have just seen, a species of information processing psychology, a way of thinking about thinking inherited largely unmodified from mainstream cognitive science. Put a little crudely, on this view the brain is the hardware in which is instantiated a functionally specified, (broadly) computational system of information retrieval and manipulation, geared towards generating behavioural outputs. That computational system is our cognitive architecture, which the evolutionary psychologist claims can be understood correctly only if one understands the adaptive problems that Darwinian selection has designed it to solve (more on this below). From this perspective, then, evolutionary psychology might be glossed as a way of Darwinizing cognitive science or, as Tooby and Cosmides (1998) themselves put it, revealing their identification of evolution with Darwinian selection, of 'evolutionizing the cognitive sciences'.

Once selectionist thinking is applied to the mind-brain, rather than to behaviours directly, there emerges a compelling solution to the worry that human social behaviour displays too much cultural diversity to permit any kind of adaptationist explanation to get a grip. Broadly speaking, this solution has two dimensions. First, it is a mundane observation that almost any useful computer program, when triggered into action, may produce any one of a range of different outputs depending on precisely which inputs it receives. So why shouldn't the same be true of our evolved psychological mechanisms? Here is the evolutionary-psychological picture: All developmentally normal human beings share a suite of innately specified psychological adaptations - selected-for internal information processing mechanisms that are robust across cultures. Any (or perhaps most) of these evolved programs may produce any one of a range of behaviours by responding differentially to varying inputs. And do the relevant inputs vary? Yes they do. Variations in the social environments in which individuals (and thus their evolved brains) are embedded produce variations in the context-sensitive real-time informational inputs available to those mechanisms. Behavioural diversity is thus purchased using a currency of inner homogeneity plus input-sensitivity. 
The second dimension of the evolutionary-psychological response to the worry about behavioural diversity appeals to a cluster of developmental issues. First we meet the developmental version of the position just described. The classic Chomskyan model of language learning posits the existence of an innately specified human-wide language acquisition device. However, as a result of that shared device being exposed to different developmental environments (different linguistic communities that provide different developmental inputs), different speakers learn and produce different languages. This model can be generalized to other innately specified psychological mechanisms. Second, evolutionary psychologists are not genetic determinists who think that innately coded-for adaptations will be present no matter what happens in the developmental environment. Whether or not a particular psychological adaptation is ultimately 'wired up' properly in a specific individual will typically depend on the presence of certain environmental triggers that, under normal circumstances, occur reliably at critical stages during development. We are all familiar with the plight of abused children whose language learning is impaired by their exposure to a linguistically impoverished environment at critical stages of their development, but there will be less dramatic examples. Finally, there may be alternative psychological adaptations available to development, alternatives that are under the control of genetic switches that initiate different developmental trajectories. For example, evolutionary psychologists argue that men and women confront divergent, sex-relative adaptive problems when it comes to finding, holding onto, and reproducing with a mate. Thus men and women instantiate different, sex-relative psychological adaptations in the mating game (more on which below). Since sex determination is under the control of a genetic switch, so are these alternative psychological architectures. Behavioural diversity (e.g. different sex-relative mating strategies) results from such switching.

The focus on inner mechanisms as adaptations, plus an observation about the typically slow pace of evolutionary change, helps the evolutionary psychologist to head off another potential worry. Here's the worry: if our psychological mechanisms are adaptations, designed with fitness advantages in mind, how come many of the behaviours that modern human beings perform are so woefully maladaptive, or at best selectively neutral? Here are three well-worn examples: given the increasing prevalence of sperm banks, adult male humans could maximize their reproductive success through a policy of widespread sperm donation, but they don't adopt such a policy; given that over-indulgence in the sugar-rich foods readily available in technologically advanced countries leads to unhealthy obesity, and thus, one might think, to a lessening of our survival and reproductive prospects, we should avoid such over-indulgence, but we don't; empirical evidence confirms that human beings have a deep-seated fear of snakes, which makes no adaptive sense at all in modern urban environments. What all this tells us is that contemporary human behaviour is not always fitness maximizing, which brings us to the first of (what I am calling) evolutionary psychology's big ideas: the environment of evolutionary adaptedness.

To see how this works we can begin by noting the all-too-often elided but in truth crucial distinction between a trait being adaptive and it being an adaptation. A trait is adaptive if its possession by some individual would, on average, bestow a fitness advantage on that 
individual. By contrast, a trait is an adaptation if its possession by some individual now is explained by the fact that it bestowed a heritable fitness advantage in ancestral environments. These two notions can come apart. Any adaptation must have been adaptive at some time in the ancestral past, but it need not be adaptive now. Thus vestigial traits may be adaptations without being adaptive. Conversely, a trait that has entered the population only very recently, meaning that selection won't have had time to act, may be adaptive now without being an adaptation.

This distinction gives us the conceptual resources, where appropriate, to decouple the present performance of a psychological mechanism with regard to fitness from the evidence one would submit in connection with its status as an adaptation. In order to pursue the latter, one needs to make sure that one has in view the selection pressures that were operative in designing the trait in question. In other words, one needs to make sure that one has in view the "composite of environmental properties of the most recent segment of a species' evolution that encompasses the period during which its modern collection of adaptations assumed their present form' (Tooby and Cosmides 1990, 388). This is what evolutionary psychologists call the environment of evolutionary adaptedness (or EEA). Of course the relevant EEA may well not be the current environment in which a trait operates. Environments sometimes change, and especially in the case of complex traits (the human brain being the most complex trait around), evolution by cumulative Darwinian selection is typically a very slow process that may lag well behind. Indeed, evolutionary psychologists argue that the last time any significant modifications were made by Darwinian selection to the human brain's functional architecture was during the Pleistocene epoch (approximately 2 million to 10 thousand years ago), when humans were hunter-gatherers. Thus the composite of selection pressures at work in the Pleistocene constitutes our environment of evolutionary adaptedness (see e.g. Crawford, 1998 for discussion). These are the adaptive problems to which our modern brains, inherited essentially unchanged from our Pleistocene hunter-gatherer ancestors, constitute evolved solutions.

Working on the assumption that one can't hope to understand what the psychological adaptations here might look like unless one understands the problems to which they constitute evolved solutions, the evolutionary psychologist's first job is to specify the adaptive problems that were present in the human EEA. According to the evolutionary psychologists, these include things like how to select a mate (Buss 1992), how to speak and understand language (Pinker and Bloom 1990, Pinker 1994), how to engage in and reason about social exchange (Cosmides and Tooby 1992), and how to explain and predict each other's behaviour (Baron-Cohen 1999).

Now we can see how evolutionary psychologists will deal with the aforementioned troubling examples of adaptive shortfall. Two are examples of adaptations that are no longer adaptive due to environmental change. Our sweet tooth was adaptive in the nutritional challenges posed by the Pleistocene, but has since been rendered maladaptive by the mass availability of refined sugar. Unfortunately selection hasn't had the time to shift the trait. A fear of snakes was adaptive given the threat posed by such creatures in the Pleistocene, but is now (presumably) selectively neutral, in that it is unlikely in the 
extreme that someone from, say, $21^{\text {st }}$ century Edinburgh, born with a 'not afraid of snakes' mutation, would do worse at survival and reproduction than her snake-fearing conspecifics. The final example is a case of a contemporary fitness-enhancing opportunity that fails to be grabbed because our psychological adaptations are meshed with our Pleistocene past: modern human males don't adopt a strategy of widespread sperm donation because our reproductive strategies are designed for Pleistocene conditions.

Evolutionary psychology's second big idea concerns the design of our evolved psychological architecture. Recall SSSM. According to that view the innate human cognitive architecture is essentially a domain-general learning and reasoning engine. By contrast, according to the evolutionary psychologists, the evolved human mind is (to use a now famous image) a kind of psychological Swiss army knife, in that it involves a large collection of specialized cognitive tools. As we have seen, evolutionary psychology is Darwinized information processing psychology, so what we have here is a picture of mind as involving a very large number of domain-specific information processing mechanisms, sometimes called modules, each of which (i) is triggered by informational inputs specific to a particular evolutionarily salient domain (e.g. choosing a mate, social exchange) and (ii) has access to internally stored information about that domain alone. Thus the Swiss army knife account of mind is sometimes glossed as the Massive Modularity Hypothesis (Samuels 1998, Sperber 1996).

Evolutionary psychology's third big idea is that behind all that diversity in human social behaviour there lurks an evolved universal human nature. In a way this point is a repackaging of claims that we've met already, but it is worth pausing to note a certain slipperiness in what exactly that evolved universal human nature might be. This slipperiness is nicely isolated by Buller (2005). The most obvious candidate for an evolved universal human nature is the suite of Darwinian modules possessed by adult human beings, but, as we know, this suite is not strictly universal, due to the existence of different developmental trajectories. So what might be strictly universal? The answer is an evolved species-wide set of genetically specified developmental programs that control processes such as genetic switching and how the emerging human phenotype responds to critical environmental triggers. It's at that level that strict universality (allegedly) holds.

Now that we have a grip on the conceptual shape of evolutionary psychology, it is worth just mentioning some of the flagship empirical claims that the approach has generated. Cosmides and Tooby (1992) argue that human beings have an evolved cognitive module specialized for spotting cheats in social exchange. Symons (1979) and Buss (1994) argue that human beings have sex-relative domain-specific mechanisms of mate preference that result in males being attracted to females who exhibit certain signs of high reproductive potential, and females being attracted to males who exhibit certain signs of high status and resource possession. Daly and Wilson (1988) argue that children reared by substitute parents (especially genetically unrelated substitute parents) are more likely to be exploited and more at risk from abuse. And Miller (2000), in a treatment that stresses sexual rather than (strict) natural selection, argues that the products of contemporary human creative intelligence, such as novels, films and jokes, need to be explained as 
human versions of the peacock's tail, in that they are elaborate ornaments that advertise the fitness of a potential mate precisely because they demonstrate that that individual has the spare capacity to use up resources on non-survival related projects.

\section{Problems for Evolutionary Psychology}

Both of evolutionary psychology's basic commitments and all three of its big ideas have been contested. In the rest of this entry I shall sample some of the lodged objections, all of which, I think, isolate open questions that are ripe for further research.

\subsection{Ultra-Darwinism}

Ultra-Darwinism is the view that almost all phenotypic traits in almost all populations of organisms are adaptations, that is, are the direct product of Darwinian selection. It does seem that evolutionary psychologists commit themselves to ultra-Darwinism about the mind, that is, to the claim that almost all features of the human cognitive architecture are psychological adaptations. Indeed, it seems to be a consequence of accepting that there is an evolutionary explanation for the human mind, and holding that evolution just means Darwinian selection (see above). So what is wrong with ultra-Darwinism? Gould (2000) argues (i) that ultra-Darwinism is on the retreat in evolutionary thinking generally, and (ii) that the human mind looks to be particularly resistant to any ultra-Darwinist treatment. The evidence for point (i) comes from (what Gould takes to be) an increasingly widespread recognition in biology that evolution is a mosaic of many different processes and phenomena, including not only Darwinian selection, but also factors such as contingency, evolutionary spandrels (traits that are not themselves selected for, but rather are by-products of selection for other traits), and punctuated equilibria (according to which the emergence of new species is not a gradual process driven by natural selection acting on geographically isolated groups in different environments, but rather involves long periods of what is essentially stasis and then moments of abrupt change).

The notion of evolutionary spandrels is particularly salient here. Gould argues that since all organisms evolve as complex and interconnected wholes, selection-driven change to one feature will typically generate non-adaptive by-products. These by-products may later be co-opted by selection to perform some function, but the existence and structure of those by-products is not explained by selection. Given that the human brain is the most complex and internally interconnected organ around, it is very likely, as Gould puts it, to be 'bursting with spandrels that establish central components of what we call human nature but that arose as non-adaptations, and therefore fall outside the compass of evolutionary psychology or any other ultra-Darwinian theory' $(2000,104)$. And that's the argument for point (ii).

Could evolutionary psychology divest itself of a perhaps indefensible ultra-Darwinism and yet remain true to its cause? I don't see why not. Indeed, evolutionary psychologists already make the point that some facets of our psychological profile will result from variation in selectively neutral features. Additionally conceding the existence of 
phenomena such as psychological spandrels would be a step towards adopting what Godfrey Smith (2001) calls explanatory adaptationism, the view that while adaptation is not ubiquitous, adaptation, and especially complex adaptation, is the central problem in evolutionary biology, and to be explained by Darwinian selection. Wearing her explanatory adaptationist hat, the evolutionary psychologist could hold that psychological adaptation is the central problem in psychology, and that it is to be explained by Darwinian selection. Other aspects of the evolutionary-psychological picture (psychological adaptations as modules, the EEA) could stay in place.

\subsection{Is the Mind an Information Processing System?}

In considering this question it is worth noting that certain prominent evolutionary psychologists commit themselves to something more specific that just some broad view of cognition as information processing. Rather, they hold that the mind is a classical rather than a connectionist computational system. One crude but effective way to state the difference between classicism and connectionism in cognitive science is to say that whereas classicism uses the abstract structure of human language as a model for the nature of mind (and thus conceives the mind as a representational system with a combinatorial syntax and semantics), connectionism uses the abstract structure of the biological brain (and thus conceives the mind as being organized into a huge number of interconnected processing units that represent the world by entering into large-scale coalitions of activation). Evolutionary psychologists have often tended to pin their colours to the classical mast because they view connectionist research, which typically starts out with a knowledge-free network that is then tuned post-natally (as it were), using generic domain-general learning algorithms, as a return to the bad old ways of SSSM. However, this commitment to the classical framework does leave the evolutionary psychologist a hostage to the fortunes of that framework, which may not be a good thing if the ongoing debate between classicists and connectionists in cognitive science (see e.g., and famously, Fodor and Pylyshyn 1988) is ever resolved in favour of the latter. Entering that complex and thorny debate would take us too far afield, and in any case there seems to be no in principle reason why one couldn't 'pre-wire' domain-specificity into a connectionist network in order to satisfy the evolutionary psychologist's conditions.

\subsection{Specifying Adaptive Problems}

The first job of the evolutionary psychologist is to specify the adaptive problems faced by our Pleistocene hunter-gatherer ancestors in the human EEA. However, some critics claim that hypotheses about how historically remote selective pressures shaped the design of minds in the distant past are hopelessly speculative, essentially untestable and thus scientifically empty (Smith et al. 2001). As Gould (2000) notes, the bands of ancient hunter-gatherers who are our target here did leave behind some tools and bones, meaning that paleoanthropologists have something to go on, but the detailed information concerning, for example, kinship relations, social structures, and the differences between the social activities of men and women that would be required to specify the relevant adaptive problems simply isn't available. Indeed, recall the claim that the human sweet tooth is an adaptation forged in the Pleistocene. Gould $(2000,100)$ points to, among other 
things, the lack of any supporting palaeontological data about ancestral feeding, as a way of dismissing that claim as 'pure guesswork in the cocktail party mode'. And even if one doesn't rule out such theorizing as overly speculative or unscientific, the fact is that paleoanthropologists and others have argued that the Pleistocene era was characterized by a highly variable set of environments and social systems, and, moreover, that some postPleistocene changes to the selective environment associated with the introduction of agriculture and urbanization have, in fact, left enough time for some more recent genetic evolution to have occurred (Smith et al. 2001). Serious worries, then, accompany the evolutionary psychologist's appeal to a human EEA.

Moreover, there is a deeper problem in the wings here, a conceptual worry about specifying adaptive problems that Sterelny and Griffiths (1999) dub the grain problem. Is, for example, choosing a mate a single adaptive problem, or is it a set of related problems, such as: choosing someone of the opposite sex, someone who shows good reproductive prospects, and someone who shows signs of being a good parent? Or at a yet finer level of description, is the problem of choosing someone with good reproductive prospects a single problem or a set of related problems, such as choosing someone who is young, who is healthy, of high status, etc.? It seems there is no final answer to any of these questions, and thus that no particular level in a selective hierarchy — or, as one might say, no individual descriptive grain - takes explanatory precedence over any other. This does not augur well for the project of specifying the particular adaptive problems to which our brains are evolved solutions.

Sterelny and Griffiths go on to suggest that the grain problem may be solved where it is possible antecedently to identify a distinct cognitive device (the adapted trait) subserving a distinct type of behaviour. In other words, our all-important level-fixing decisions may be constrained by the prior identification of distinct cognitive devices. Unfortunately there is good reason to be suspicious of Sterelny and Griffiths' strategy, because the grain problem comes in not just one but two dimensions, the second of which concerns the cognitive devices themselves (Atkinson and Wheeler 2004). Thus, are the large-scale neural pathways in the human visual system distinct cognitive devices, or should we descend to a lower level of description and the 30 or so functionally distinct areas of visual cortex? Of course, if we knew what the adaptive problem was, we could determine the right architectural level, but that takes us back to the first dimension of the problem. Still, there might be a way out of the grain problem. Perhaps the evolutionary psychologist can avail herself of an established methodology already at work elsewhere in evolutionary theorizing about the mind/brain, a methodology takes the grain problem on the chin by accepting that at least sometimes there will be equally good evolutionary stories to tell at different levels of organization, stories that must at least be compatible and ideally mutually supporting. (For more on how this story might go, see Atkinson and Wheeler (2004).)

\subsection{Are there any Good Arguments for Massive Modularity?}

In arguing for massive modularity, evolutionary psychologists sometimes develop inprinciple arguments which aim to establish that domain-general mechanisms on their 
own, i.e. without assistance from domains-specific mechanisms, would not be able to solve the adaptive problems faced by our hunter-gatherer ancestors (see e.g. Tooby and Cosmides 1992, 102-112). Perhaps the most powerful of these arguments goes like this: In part, the domain-generality of an inner mechanism would be guaranteed by that mechanism having free access to the overall stock of beliefs possessed by some system, such that it could operate in a large number of domains. However, before such a mechanism could determine a contextually appropriate response to some input, it would first need to consider all the beliefs to which it had access, in order to work out which of them were, in fact, relevant. (This will be familiar to some readers as the frame problem from artificial intelligence.) Tooby and Cosmides' point is that the number of beliefs that a domain-general mechanism would need to consider in this way would always be prohibitively large, leading to a crippling computational load and thus adaptive impotence. And as long as we preserve a commitment to domain-general mechanisms, this worry won't be deflected by some appeal to stored relevancy heuristics that determine which of the system's beliefs is relevant in any particular scenario, since this just ushers in another Herculean search problem - finding the right relevancy heuristic.

It is at least plausible that the massive modularity view avoids this difficulty. In any context of action, the domain-specific mechanism that is appropriately activated will (as a direct consequence of evolutionary design) have access to no more than a highly restricted subset of the system's entire range of beliefs. Moreover, that subset will include just those beliefs that are relevant to the adaptive scenario in which the system finds itself. Therefore the kind of unmanageable search space that stymies the progress of any domain-general mechanism is simply never established. Putting aside a nagging worry about just how the right module gets activated here (presumably not by some domaingeneral module-selection mechanism!), Tooby and Cosmides' argument in favour of domain-specificity remains much too swift (Wheeler and Atkinson 2001, Mameli 2001, Atkinson and Wheeler 2004). That's because the deep reason why the modular architecture avoids the specified problem is that the body of information to which each module has access is restricted in adaptively useful ways. However, now consider an intrinsically domain-general psychological mechanism that, through the opportunistic process of design by selection, has been allowed access only to a restricted body of information. That mechanism would have the desired property too. If this is right, then the question of whether or not the mechanisms of mind are domain-specific or domaingeneral in character remains open.

Perhaps the case can be re-mounted as a matter of efficiency rather than all-or-nothing achievement. Thus Cosmides and Tooby claim that domain-specific cognitive mechanisms ... can be expected to systematically outperform (and hence preclude or replace) more general mechanisms' (Cosmides and Tooby 1994, 89). The idea is that, in the human EEA, any domain-general mechanism in the population will typically have been systematically outperformed by any competing domain-specific mechanism. Thus it is the latter kind of mechanism that will have been selected for. However, Samuels (1998) points out that Cosmides and Tooby's arguments establish only that systems with domain-specific features of some kind, and that includes a domain-general mechanism 
with access to domain-specific information, will outperform competing systems without such features.

At this juncture it is worth noting two things. First, from the perspective of a broader evolutionarily-informed approach to psychology, the domain-specific versus domaingeneral issue has a life beyond the battle over massive modularity. Indeed, striking a balance between domain-specificity and domain-generality may itself be an adaptive problem that selection has solved. For example, Gigerenzer and Todd (1999) defend the idea that human rationality is characterized largely by the deployment of fast and frugal heuristics, simple reasoning strategies that exploit the structure of environmental information to make adaptively beneficial decisions. Such heuristics are specific to particular information structures, but not so specific to particular environments that they don't generalize to previously unencountered environments, which indicates that some degree of domain-generality is in play.

The second thing to note is that a more robust domain-generality than this need not be anathema to evolutionary thinking in psychology. For example, drawing on Boyd and Richerson's (1985) dual inheritance model, which stresses cultural as well as genetic transmission in evolution, Coultas (2004) provides experimental evidence that individual human beings have an essentially domain-general tendency to conform in social groups, a tendency that can be adaptive for the individual when information-gathering by that individual would be costly. And Tomasello (1999), in a treatment that also stresses dual inheritance, argues that evolution has endowed us with a set of basic cognitive capacities, including shared attention and the imitation of other humans' behaviours and intentions, that allow us to take developmental advantage of a kind of accumulated species-specific knowledge made available through human cultural environments. At the heart of this process, and the capacity that sets human beings apart from other species, is our ability to identify intentions in others. It's this uniquely human, essentially domain-general ability, argues Tomasello, that allows us to build on foundational capacities that we share with other animals (such as the capacities for tool-use and signalling), in order to become vastly more sophisticated thinkers in specific domains (e.g. vastly more sophisticated tool-users and signallers) than have our evolutionary cousins. (For criticisms of this idea, see e.g. Hauser (2000).)

\subsection{Is there an Evolved Human Nature?}

The evolutionary psychologist's notion of an evolved human nature is that of a specieswide set of genetically specified developmental programs that orchestrate the journey from genotype to phenotype. A maturing human being embedded in a normal developmental environment will thus end up with a particular, species-wide set of cognitive modules (allowing for some branching pathways, e.g. between the sexes). As Buller (2005) notes, this idea appears to depend on what Sober (1980) calls the natural state model in biology. With its roots in Aristotle, this model holds that diversity and variation among organisms of the same species are deviations from the species-relative natural state of those organisms, deviations caused by the operation of interfering forces, such as those in abnormal developmental environments. Thus the natural state model 
identifies the natural phenotype (e.g. the evolved human nature) by identifying a privileged developmental environment, the natural one. However, the natural state model, and thus (ironically) evolutionary psychology, is at root in conflict with contemporary neo-Darwinian evolutionary biology, which treats the biological realm as a place in which individual variation is conceived as the fundamental way of things, rather than as the product of interfering forces that deflect individuals from a path towards a state of species-wide uniformity. Thus contemporary evolutionary theory thinks in terms of norms of reaction (genotype-phenotype mappings in particular environments), with each phenotypic outcomes, and thus each of the associated developmental environments, conceptualized as being on an equal footing.

The evolutionary psychologist, like any natural state theorist, needs to privilege one of the possible developmental environments as the 'natural' one. It might seem that the EEA might be the ticket here - an environment that counts as privileged because it is the home of the adaptive problems to which our psychological adaptations constitute solutions, and which thus might be considered our natural environment. But this suggestion runs bang up against the aforementioned worry that the evolutionary psychologist mistakes Darwinian selection for the whole of evolution. As Buller $(2005,436)$ notes, 'adaptation is just one process among many in evolution, and nothing in evolutionary theory privileges the process of adaptation over other processes [such as genetic drift or migration in and out of populations] by considering it more natural than other processes'. In closing it is worth noting that this is a mistake that Darwin himself didn't make. So the much trumpeted Darwinization of psychology as championed by evolutionary psychology (narrowly conceived) may not be a genuine Darwinization at all. That would be achieved by a more pluralistic, multi-faceted and correspondingly richer understanding of evolution, and thus of the evolved character of the human mind.

Michael Wheeler

Department of Philosophy

University of Stirling

UK

\section{References}

Atkinson, A., and Wheeler, M. (2004). The grain of domains: The evolutionarypsychological case against domain-general cognition, Mind and Language, 19:2, 147-176.

Baron-Cohen, S. (1999). The evolution of a theory of mind. In M.C. Corballis and S.E.G. Lea (Eds), The Descent of Mind: Psychological Perspectives on Hominid Evolution, (pp. 261-77). Oxford: Oxford University Press.

Boyd, R. and Richerson, P.J. (1985). Culture and the Evolutionary Process. Chicago IL: University of Chicago Press. 
Buller, D. J. (2005). Adapting Minds: Evolutionary Psychology and the Persistent Quest for Human Nature. Cambridge, MA: MIT Press.

Buss, D.M. (1992). Mate preference mechanisms: Consequences for partner choice and intrasexual competition. In J.H. Barkow, L. Cosmides and J. Tooby (Eds), The Adapted Mind: Evolutionary Psychology and the Generation of Culture (pp. 24966). New York: Oxford University Press.

Buss, D.M. (1994). The Evolution of Desire: Strategies of Human Mating. New York: Basic Books.

Cosmides, L., and Tooby, J. (1987). From evolution to behavior: Evolutionary psychology as the missing link. In J. Dupre (Ed), The Latest on the Best: Essays on Evolution and Optimality (pp. 227-306). Cambridge, MA: MIT Press.

Cosmides, L. and Tooby, J. (1992). Cognitive adaptations for social exchange. In J.H. Barkow, L. Cosmides and J. Tooby (Eds), The Adapted Mind: Evolutionary Psychology and the Generation of Culture (pp. 163-228). New York: Oxford University Press.

Cosmides, L. and Tooby, J. (1994). Origins of domain specificity: The evolution of functional organization. In L.A. Hirschfeld and S.A. Gelman (Eds), Mapping the Mind: Domain Specificity in Cognition and Culture (pp. 85-116). Cambridge and New York: Cambridge University Press.

Coultas, J.C. (2004). When in Rome... An evolutionary perspective on conformity. Group Processes and Intergroup Relations. 7(4), 317-331.

Crawford, C. (1998). Environments and adaptations: Then and now. In C. Crawford and D.L. Krebs (Eds), Handbook of Evolutionary Psychology: Ideas, Issues, and Applications (pp. 275-302). Mahwah, NJ: Lawrence Erlbaum.

Daly, M. and Wilson, M. (1988). Homicide. New York: Aldine de Gruyter.

Fodor, J.A., Pylyshyn, Z., (1988). Connectionism and cognitive architecture: A critical analysis. Cognition 28, 3-71.

Gigerenzer, G., Todd, P.M. and the ABC Research Group. (1999). Simple Heuristics That Make Us Smart. Oxford: Oxford University Press.

Godfrey-Smith, P. (2001). Three kinds of adaptationism. In S. H. Orzack and E. Sober (Eds), Adaptationism and Optimality (pp. 335-357). Cambridge: Cambridge University Press. 
Gould, S. J. (2000). More things in heaven and earth. In H. Rose and S. Rose (Eds) Alas Poor Darwin: Arguments Against Evolutionary Psychology (pp. 101-126). New York: Harmony Books.

Hauser, M.D. (2000). Et tu Homo sapiens? (review of Tomasello 1999). Science 288: $5467,816-817$.

Mameli, M. (2001). Modules and mindreaders (multiple book review). Biology and Philosophy. 16, 377-33.

Miller, G. (2000). The Mating Mind: How Sexual Choice Shaped the Evolution of Human Nature. London: William Heinemann.

Pinker, S. (1994). The Language Instinct. London: Allen Lane/ Penguin.

Pinker, S. and Bloom, P. (1990). Natural language and natural selection. Behavioral and Brain Sciences, 13, 707-27.

Samuels, R. (1998). Evolutionary psychology and the massive modularity hypothesis. British Journal for the Philosophy of Science, 49, 575-602.

Smith, E.A., Borgerhoff, Mulder, M. and Hill, J. (2001). Controversies in the evolutionary social sciences: A guide for the perplexed. Trends in Ecology and Evolution, 16:3, 128-135.

Sober, E. (1980). Evolution, population thinking, and essentialism. Philosophy of Science 47, 350-383.

Sperber, D. (1996). Explaining Culture: A Naturalistic Approach. Oxford: Basil Blackwell.

Symons, D. (1979). The Evolution of Human Sexuality. Oxford: Oxford University Press.

Thornhill, R. and Thornhill, N. (1992). The evolutionary psychology of men's coercive sexuality. Behavioral and Brain Sciences, 15, 363-421.

Tomasello, M. (2000). The Cultural Origins of Human Cognition. Cambridge, MA: Harvard University Press.

Tooby, J. and Cosmides, L. (1990). The past explains the present: Emotional adaptations and the structure of ancestral environments. Ethology and Sociobiology 11: 375424.

Tooby, J. and Cosmides, L. (1992). The psychological foundations of culture. In J.H. Barkow, L. Cosmides and J. Tooby (Eds), The Adapted Mind: Evolutionary Psychology and the Generation of Culture (pp. 19-136). New York: Oxford 
University Press.

Tooby, J. and Cosmides, L. (1998). Evolutionizing the cognitive sciences: A reply to Shapiro and Epstein. Mind and Language, 13, 195-204.

Wheeler, M., and Atkinson, A., (2001). Domains, brains and evolution, in Walsh, D. M. (Ed), Naturalism, Evolution and Mind (pp. 239-66). Cambridge: Cambridge University Press.

Wilson, E. (1975). Sociobiology: the New Synthesis. Cambridge, MA: Harvard University Press

\section{Suggested Readings}

J.H. Barkow, J.H., Cosmides, L. and Tooby, J. (Eds) (1992). The Adapted Mind: Evolutionary Psychology and the Generation of Culture. New York: Oxford University Press.

Buller, D. J. (2005). Adapting Minds: Evolutionary Psychology and the Persistent Quest for Human Nature. Cambridge, MA: MIT Press.

Buss, D.M (2005). The Handbook of Evolutionary Psychology. Hoboken, NJ: Wiley.

Crawford, C. and Krebs, D.L. (Eds), (1998). Handbook of Evolutionary Psychology: Ideas, Issues, and Applications. Mahwah, NJ: Lawrence Erlbaum.

Gigerenzer, G., Todd, P.M. and the ABC Research Group. (1999). Simple Heuristics That Make Us Smart. Oxford: Oxford University Press.

Miller, G. (2000). The Mating Mind: How Sexual Choice Shaped the Evolution of Human Nature. London: William Heinemann.

Pinker, S. (1997). How the Mind Works. New York: Norton.

Rose, H. and Rose, S. (Eds) (2000). Alas Poor Darwin: Arguments Against Evolutionary Psychology. New York: Harmony Books.

Tomasello, M. (2000). The Cultural Origins of Human Cognition. Cambridge, MA: Harvard University Press. 